\title{
Silencing Lin28 promotes apoptosis in colorectal cancer cells by upregulating let-7c targeting of antiapoptotic BCL2L1
}

\author{
HAOGANG ZHANG ${ }^{1}$, YAGUANG ZONG ${ }^{1}$, GONGCAI QIU ${ }^{1}$, \\ RUICHUN JIA ${ }^{2}$, XUNZHENG XU ${ }^{1}$, FUJING WANG ${ }^{1}$ and DEQUAN WU ${ }^{1}$ \\ Departments of ${ }^{1}$ General Surgery and ${ }^{2}$ Blood Transfusion, \\ The Second Affiliated Hospital of Harbin Medical University, Harbin, Heilongjiang 150081, P.R. China
}

Received December 6, 2016; Accepted October 17, 2017

DOI: $10.3892 / \mathrm{mmr} .2018 .8483$

\begin{abstract}
Colorectal cancer (CRC) remains a primary contributor to cancer-associated mortality. The Lin28/let-7 axis has previously been verified to participate in numerous pathophysiological processes involved in CRC. However, the potential roles and underlying mechanisms of this axis in apoptosis during CRC remain to be fully elucidated. The present study aimed to evaluate the role and reveal the molecular mechanisms of the Lin28/let-7 axis in the apoptosis of CRC cells. An MTT assay was conducted to assess the cell viability of HCT116 and HT29 CRC cells, and caspase-3 activity was analyzed to measure the apoptosis of CRC cells. Western blotting and reverse transcription-quantitative polymerase chain reaction were performed to examine the expression of Lin28, B-cell lymphoma 2 (Bcl-2), Bcl-2-associated X protein, Bcl-2-like 1 (BCL2L1) and let-7c. The present study demonstrated that Lin28 was upregulated whereas let-7c was downregulated in CRC tissues and cell lines compared with normal tissues and NCM460 normal colon epithelial cells, respectively. Forced overexpression of let-7c promoted apoptosis in CRC cells, which was at least partially mediated via the targeting of BCL2L1. Furthermore, knockdown of Lin28 decreased viability and promoted apoptosis in CRC cells, whereas this effect was attenuated by let-7c inhibition. The findings of the present study suggest the involvement of the Lin28/let-7c axis in apoptosis during $\mathrm{CRC}$, and indicate the potential role of this pathway as a novel therapeutic target in CRC.
\end{abstract}

\section{Introduction}

Colorectal cancer (CRC) is the second most commonly diagnosed cancer in females and the third in males worldwide, with

Correspondence to: Dr Dequan Wu, Department of General Surgery, The Second Affiliated Hospital of Harbin Medical University, 246 Xuefu Road, Harbin, Heilongjiang 150081, P.R. China

E-mail: wudequan2017@126.com

Key words: colorectal cancer, Lin28, let-7, Bcl-2-like 1, apoptosis $\sim 1.4$ million new cases and 700,000 mortalities in 2012 (1). It is estimated that its incidence will increase by $60 \%$ by 2030 (2). Although various drug treatments, including active chemotherapeutic drugs (e.g. fluoropyrimidines and oxaliplatin) and targeted drugs (e.g. bevacizumab and cetuximab), have been applied to reduce the incidence and mortality of $\mathrm{CRC}$, the efficacy of these drugs in CRC remains limited $(3,4)$. Phytochemicals are expected to become a novel option for CRC prevention and treatment (5). However, cancer is a complex disorder associated with defects in multiple signaling pathways that confer resistance to apoptosis.

Increasing evidence indicates that microRNAs (miRNAs/ miRs) contribute to the initiation and progression of various types of cancer, including liver, lung and colorectal cancers $(6,7)$. As one of the first miRNAs to be described, let-7 has been demonstrated to participate in the development of cancer (8). Furthermore, the RNA-binding protein Lin28 is an emerging oncogenic driver which acts by restraining the biogenesis of let-7 (9). King et al (10) found that Lin28B is increased in CRC patients and promotes the progression and metastasis of CRC. In addition, a recent study by Tu et al (11) demonstrated that the Lin28/let-7 axis promotes invasive intestinal adenocarcinoma in murine models by cooperating with the Wnt pathway. However, the role of the Lin28/let-7 loop in the apoptosis of CRC cells is not well understood.

The primary objective of the present study was to examine whether Lin28/let-7 is involved in the apoptosis of CRC cells and elucidate the underlying molecular mechanisms of this. It was revealed that forced expression of let-7c, or silencing of Lin28, led to reduced cell viability and increased apoptosis in CRC cells, indicating the potential for this loop as a novel therapeutic target for CRC.

\section{Materials and methods}

Patient samples. Cancer tissue samples and adjacent normal tissues were obtained from 10 patients (6 male and 4 female; mean age, 55.3 \pm 5.7 years) with CRC who underwent surgical resection of primary tumors at the Second Affiliated Hospital of Harbin Medical University (Harbin, China) between 2016 and 2016. The patients did not receive radiotherapy or chemotherapy prior to resection. The specimens were immediately snap-frozen in liquid nitrogen and stored at $-80^{\circ} \mathrm{C}$ until use. 
All procedures were approved by the Ethics Committee for the Use of Human Samples of Harbin Medical University. Written informed consent was obtained from all patients.

Immunohistochemistry and quantification. Formalin-fixed, paraffin-embedded tissue sections (thickness, $5 \mu \mathrm{m}$ ) were treated with xylene followed by a graded alcohol series, and antigen retrieval was performed using $0.01 \mathrm{M}$ citrate buffer. Hydrogen peroxide was used for blocking of endogenous peroxidase activity. Tissue sections were then treated with goat serum for $20 \mathrm{~min}$. Subsequently, antibodies against Lin28 (\#sc-293120; Santa Cruz Biotechnology, Inc., Santa Cruz, CA, USA; dilution, 1:100) were incubated with each section at $4^{\circ} \mathrm{C}$ overnight. An EliVision ${ }^{\mathrm{TM}}$ Plus kit and a DAB kit (both from Beyotime Institute of Biotechnology, Haimen, China) were used to detect the bound primary antibodies. Staining was performed according to manufacturer's protocol. All tumor slides were examined under a light microscope by two independent pathologists. Lin28 signals in the tissues were visually quantified using a scoring system in which the score for the intensity of signal ( 0 , no signal; 1 , weak signal; 2 , intermediate signal; and 3, strong signal) was multiplied by the score for the percentage of positive cells $(0,0 \% ; 1,<25 \% ; 2,25-50 \%$; and $3,>50 \%$ ) to produce an overall score ranging from 0 to 9 .

Cell culture and treatments. NCM460 normal human colonic epithelial cells and the human CRC cell lines HCT116 and HT29 were obtained from the Shanghai Cell Bank of the Chinese Academy of Sciences (Shanghai, China). Cells were cultured in RPMI-1640 medium (Hyclone; GE Healthcare Life Sciences, Logan, UT, USA) supplemented with $10 \%$ fetal bovine serum (Hyclone; GE Healthcare Life Sciences), at $37^{\circ} \mathrm{C}$ in a humidified atmosphere of $95 \%$ air and $5 \% \mathrm{CO}_{2}$. Cells transfected with siRNAs, let-7c or AMO-let-7c (Shanghai GenePharma, Co., Ltd, Shanghai, China) were collected at $48 \mathrm{~h}$ post-transfection for further measurements.

Transfection. Prior to transfection, HCT116 or HT29 cells were grown in $25-\mathrm{cm}^{2}$ cell culture flasks with $4 \mathrm{ml}$ medium. The let-7c mimics or AMO-let-7c (let-7c antisense oligonucleotides), or their respective negative controls (NCs), as well as $10 \mu \mathrm{l}$ Lipofectamine 2000 (Invitrogen; Thermo Fisher Scientific, Inc., Waltham, MA, USA) were separately mixed with $500 \mu$ l of Opti-MEM ${ }^{\circledR}$ I Reduced Serum Medium (Gibco; Thermo Fisher Scientific, Inc.) for $5 \mathrm{~min}$. Subsequently, the two mixtures were combined and incubated for $20 \mathrm{~min}$ at room temperature. The final concentration of miRNAs was $100 \mu \mathrm{M}$. The Lipofectamine-miRNA mixtures were then added to the cells, which were incubated at $37^{\circ} \mathrm{C}$ for $36 \mathrm{~h}$ prior to further experiments. The control cells underwent the same transfection procedures without nucleic acid. The NC for let-7c overexpression was a disordered sequence of let-7c. The transfection protocol for the siRNAs $(20 \mathrm{nM})$ was the same as that for miRNAs. The sequences of the RNAs and controls were as follows: let-7c mimics, 5'-UGAGGUAGUAGGUUG UAUGGUU-3'; AMO-let-7c, 5'-AACCAUACA ACCUAC UACCUCA-3; let-7c NC, 5'-UUCUCCGAACGUGUCACG UTT-3' (sense) and 5'-ACGUGACACGUUCGGAGAATT-3' (anti-sense); AMO-NC (NC for AMO-let-7c), 5'-CAGUAC UUUUGUGUAGUACAA-3'; si-Lin28 \#1, 5'-GGAGACAGG
UGCUACAACUUU-3'; si-Lin28 \#2: 5'-UGACGUAUCUUG UGCGUUUUU-3'; si-Lin28 \#3: 5'-AAAUGUGUCUCACGG GUUUUU-3'; scrambled siRNA, 5'-UGCGGAUUCUAU CUGUAU-3'.

MTT cell viability assay. HCT116 or HT29 cells were seeded in 96-well culture plates in $200 \mu \mathrm{l}$ medium with $1 \times 10^{4}$ cells/well, and incubated at $37^{\circ} \mathrm{C}$ with $5 \% \mathrm{CO}_{2}$. Following the transfection of siRNAs or miRNAs, an MTT assay (Amresco, LLC, Solon, OH, USA) was performed. Briefly, $20 \mu \mathrm{l}$ of MTT solution $(5 \mathrm{mg} / \mathrm{ml})$ was added to each well, and the cells were continuously incubated for $4 \mathrm{~h}$. Following removal of cell culture medium, formazan crystals were dissolved in $150 \mu \mathrm{l}$ DMSO. The optical density (OD) of each wells was measured with a microplate reader (BioTek Instruments, Inc., Winooski, VT, USA) at $490 \mathrm{~nm}$.

RNA isolation and quantification of Lin28 and let-7c. Total RNA was extracted from CRC tissue samples or HCT116 or HT29 cells by TRIzol ${ }^{\circledR}$ reagent (Invitrogen; Thermo Fisher Scientific, Inc.) following the manufacturer's protocol. Total RNA $(0.5 \mu \mathrm{g})$ was then reverse transcribed using a High-Capacity cDNA Reverse Transcription Kit (Applied Biosystems; Thermo Fisher Scientific, Inc.) to obtain cDNA. The temperature protocol was as follows: $25^{\circ} \mathrm{C}$ for $10 \mathrm{~min}$, $37^{\circ} \mathrm{C}$ for $120 \mathrm{~min}, 85^{\circ} \mathrm{C}$ for $5 \mathrm{~min}$, and hold at $4^{\circ} \mathrm{C}$. The RNA level of Lin28 was determined using a SYBR Green I incorporation method on an ABI 7500 Fast Real-Time PCR system (Applied Biosystems; Thermo Fisher Scientific, Inc.), with GAPDH as an internal control. The let-7c level was measured using a mirVana ${ }^{\mathrm{TM}}$ qRT PCR miRNA Detection Kit (Ambion; Thermo Fisher Scientific, Inc.), following the method described by Liang et al (12). The protocol was as follows: $95^{\circ} \mathrm{C}$ for $10 \mathrm{~min}$; followed by 40 cycles of $95^{\circ} \mathrm{C}$ for $15 \mathrm{sec}, 60^{\circ} \mathrm{C}$ for $30 \mathrm{sec}$, and $72^{\circ} \mathrm{C}$ for $30 \mathrm{sec}$. The primers for qPCR were as follows: let-7c forward, 5'-GGGAGAGGTAGT AGGTTG-3'; and let-7c reverse, 5'-TGGAGTCGGCAATTG CAC-3'; U6 forward, 5'-GCTTCGGCAGCACATATACTA AAAT-3'; U6 reverse, 5'-CGCTTCACGAATTTGCGTGTC AT-3'; Lin28 forward, 5'-TCTACCTCCTCAGCCAAAGA-3'; Lin28 reverse, 5'-TGGGATTCTGCTTCCTGTCT-3'; GAPDH forward, 5'-GGGGCTCTCTGCTCCTCCCTG-3'; and GAPDH reverse, 5'-CGGCCAAATCCGTTCACACCG-3'. Variations in the expression of let-7c between different RNA samples were calculated after normalization to U6. The data were analyzed using the $2^{-\Delta \Delta \mathrm{Cq}}$ method (13); for the cell lines, cells treated with Lipofectamine 2000 only were used as a control group. The experiment was repeated independently five times.

Western blot. For western blot analysis, total protein samples were extracted from HCT116 or HT29 cells. Cells were lysed with radioimmunoprecipitation assay (RIPA)lysis buffer (Beyotime Institute of Biotechnology, Haimen, China). Total protein was quantified using a BCA Assay kit (Beyotime Institute of Biotechnology) according to the manufacturer's protocol. Total protein samples $(50 \mu \mathrm{g})$ were separated on a $15 \%$ SDS-polyacrylamide gel. After electrophoretic transfer of the proteins to a pure nitrocellulose blotting membrane, the blots were blocked with 5\% non-fat dry milk (Beyotime 

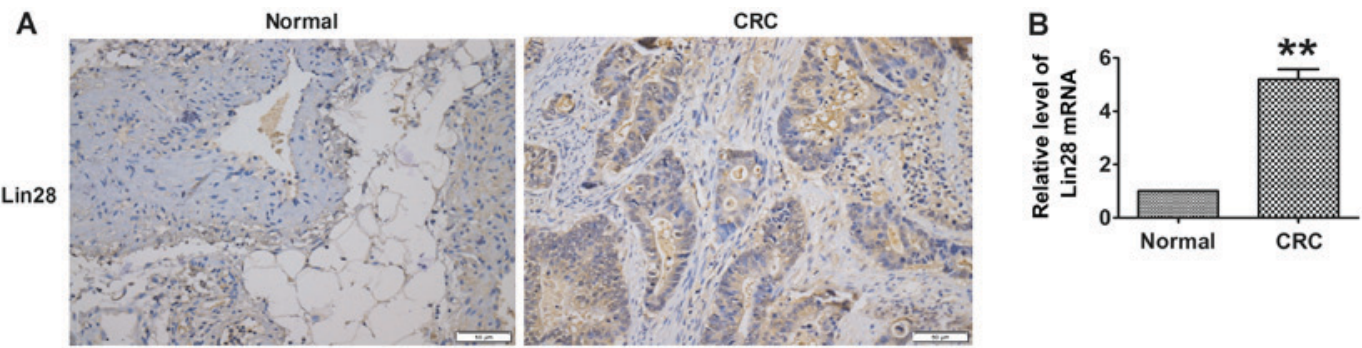

E

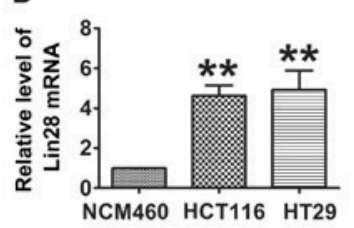

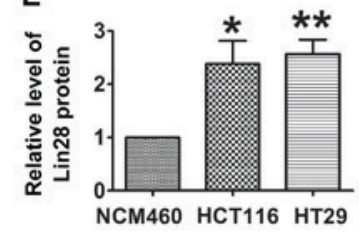

Lin28

GAPDH
$\mathrm{F}$

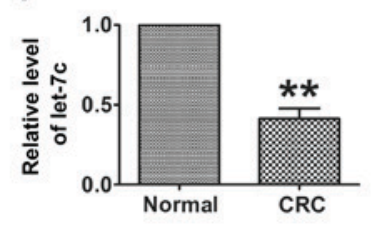

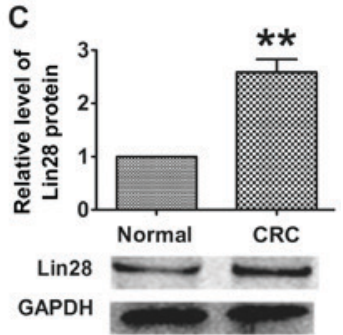

G

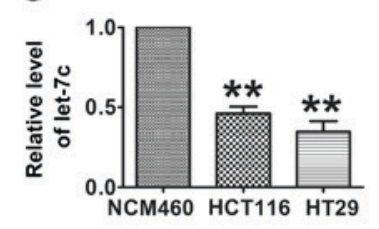

Figure 1. Dysregulation of Lin28/let-7c axis is observed in CRC patient tissues and cell lines. (A) Immunohistochemical staining of Lin28 in normal and CRC tissues from patients (scale bar, $50 \mu \mathrm{m}$ ). (B-E) mRNA and protein levels of Lin28 were increased in (B and C) CRC patient tissues and (D and E) CRC cell lines compared with normal tissues and NCM460 normal colon epithelial cells, respectively. (F and G) Let-7c levels were decreased in (F) patients with CRC and (G) CRC cell lines compared with normal tissues and NCM460 normal colon epithelial cells, respectively. Patient tissues, $\mathrm{n}=10$; cell lines, $\mathrm{n}=5$ independent experiments. ${ }^{*} \mathrm{P}<0.05$ and ${ }^{* *} \mathrm{P}<0.01$ vs. normal tissues or NCM460 cells). CRC, colorectal cancer.

Institute of Biotechnology) for $2 \mathrm{~h}$ at room temperature, then incubated overnight at $4^{\circ} \mathrm{C}$ with rabbit polyclonal antibodies against B-cell lymphoma 2 (Bcl-2) (\#3498; Cell Signaling Technology, Inc., Danvers, MA, USA; dilution, 1:800), Bcl-2-associated X protein (Bax) (\#14796; Cell Signaling Technology, Inc.; dilution, 1:800), Bcl-2-like 1 (BCL2L1) (\#10783-1-AP; ProteinTech, Wuhan, China; dilution, 1:200) and Lin28 (\#sc-293120; Santa Cruz Biotechnology, Inc., Santa Cruz, CA, USA; dilution, 1:200), and an anti-GAPDH antibody (\#KC-5G4; Kangchen Biotech, Shanghai, China; dilution, 1:2,000), which was used as an internal control. The blots were subsequently incubated with a DyLight 800-conjugated secondary antibody (\#5151; Cell Signaling Technology, Inc.; dilution, 1:1,000) for $2 \mathrm{~h}$ at room temperature, and bands were detected using an Odyssey Infrared Imaging System and analyzed using Odyssey software v1.2 (Infrared Imaging System LI-COR Biosciences). The bands were quantified by measuring the band intensity for each group.

Caspase-3 activity assay. HCT116 and HT29 cells were lysed in $50 \mu \mathrm{l}$ of ice-cold RIPA lysis buffer for $30 \mathrm{~min}$. The caspase- 3 activity assay kit was obtained from the Beyotime Institute of Biotechnology. The lysates were centrifuged at $16,000 \mathrm{x} g$ for $15 \mathrm{~min}$ at $4^{\circ} \mathrm{C}$. The fluorogenic substrates for caspase-3 were labeled with the p-nitroaniline (pNA). The enzyme activity was determined by monitoring the fluorescence produced by free pNA using a spectrofluorophotometer (RF-5301 PC; Shimadzu Corporation, Kyoto, Japan) at $405 \mathrm{~nm}$. Caspase-3 activity was expressed as micromoles of pNA liberated per minute per microgram of protein, following determination of total protein concentration using a BCA Assay kit (Beyotime Institute of Biotechnology) according to the manufacturer's protocol.

Luciferase reporter assays. The TargetScan database (http:// www.targetscan.org/vert_71/) was utilized to determine the direct target of let-7c. The BCL2L1 3'-UTR containing the conserved let-7c-binding sites was synthesized by Invitrogen and subcloned using the SacI and HindIII sites downstream of the luciferase gene in a pMIR-REPORT Luciferase vector (Promega Corporation, Madison, WI, USA). The luciferase vector (100 ng) containing the 3'-UTR was cotransfected with let-7c mimics into HEK 293 cells using Lipofectamine 2000. As an internal control, $10 \mathrm{ng}$ of Renilla luciferase reporters were also included. At $36 \mathrm{~h}$ after transfection, the cells were collected and dual luciferase activities were measured by a luminometer (Promega Corporation) according to the manufacturer's instructions.

Statistical analysis. All data are presented as the mean \pm standard error of the mean. A Student's t-test was used for two-group comparisons, and a one-way ANOVA followed by a Bonferroni test for multiple comparisons was used for comparisons between three or more groups. Two-tailed $\mathrm{P}<0.05$ was considered to indicate a statistically significant difference.

\section{Results}

Dysregulation of the Lin28/let-7 axis in patients with CRC and in CRC cells. To investigate whether the Lin28/let-7 axis participates in the process of CRC, immunohistochemistry, RT-qPCR and western blot analyses were used to detect the levels of Lin28/let-7 in patient-derived CRC tissues and in CRC cells. Immunohistochemistry revealed that Lin28, which was mainly located in the cytoplasm of the cells, was overexpressed in CRC tissues compared with adjacent normal colorectal tissues $(7.22 \pm 0.94$ vs $2.11 \pm 0.61, \mathrm{P}<0.05$; Fig. $1 \mathrm{~A})$. In addition, as shown in Fig. $1 \mathrm{~B}$ and $\mathrm{C}$, the expression of Lin28 was significantly increased at the mRNA and protein levels in the CRC samples. In accord with the data from patients, the mRNA and protein levels of Lin28 were revealed to be 

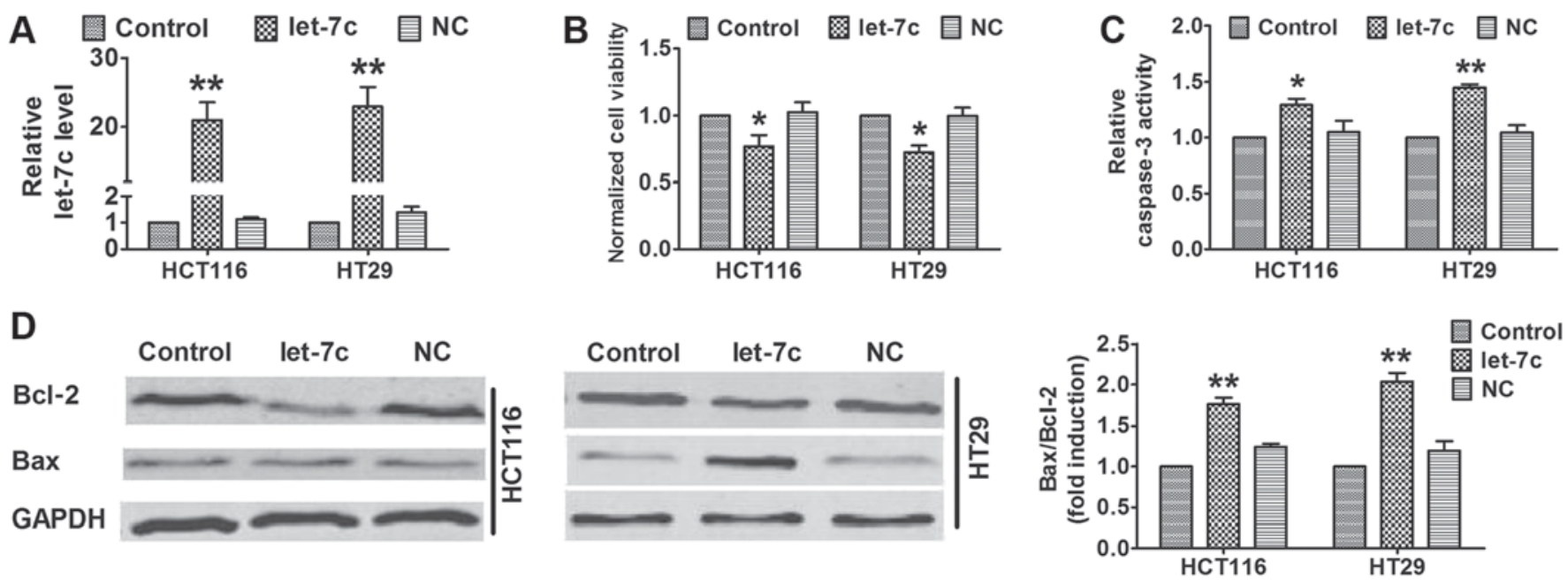

Figure 2. Enhanced expression of let-7c leads to apoptosis in CRC cells. (A) Transfection of let-7c mimics into CRC cells increased its expression. (B) Overexpression of let-7c decreased the viability of CRC cells, as assessed by MTT assay. (C) Forced expression of let-7c led to the upregulation of caspase-3 activity in CRC cells. (D) Forced expression of let- $7 \mathrm{c}$ caused dysregulation of $\mathrm{Bcl}-2$ and Bax proteins, as determined by western blotting. $\mathrm{n}=5$ independent experiments; $\mathrm{P}<0.05$ and ${ }^{* *} \mathrm{P}<0.01$ vs. control group. $\mathrm{CRC}$, colorectal cancer; $\mathrm{NC}$, negative control (mimic control); Bcl-2, B-cell lymphoma 2; Bax, Bcl-2-associated X protein.

markedly increased in CRC cells compared with NCM460 normal colonic epithelial cells (Fig. 1D and E). Furthermore, as a downstream factor of Lin28, the expression of let-7c was markedly decreased in the CRC patient tissues and in CRC cells compared with the respective normal controls (Fig. 1F and $\mathrm{G})$. These results suggest that the dysregulation of the Lin28/let-7c axis may contribute to the process of CRC.

Forced expression of let-7c induces apoptosis in cultured CRC cells. To determine the role of let-7c in the process of CRC, let-7c miRNA mimics were transfected into CRC cells (Fig. 2A). As shown in Fig. 2B, cell viability was decreased in HCT116 and HT29 cells following let-7c mimic transfection. In addition, overexpression of let-7c led to activation of caspase-3 activity in HCT116 and HT29 cells (Fig. 2C). Furthermore, western blot analysis revealed altered $\mathrm{Bcl}-2$ and Bax protein levels in let-7c mimic-transfected CRC cells (Fig. 2D). These results suggest that enhanced expression of let-7c could induce apoptosis in CRC cells.

Let-7c regulates BCL $2 L 1$ in a post-transcriptional manner. TargetScan, an online miRNA target prediction database, was employed to determine the direct target of let-7c. As depicted in Fig. 3A, let-7c has seed sequence complementary with the binding site in the $3^{\prime}$ untranslated region (UTR) of BCL2L1, an antiapoptotic factor. A luciferase assay showed that co-transfection of let-7c mimics with a luciferase reporter vector carrying a portion of the wild-type human BCL2L1 3'UTR led to a significant decrease in luciferase activities compared with transfection with the luciferase vector alone (control group), whereas transfection with the NC had no significant effect (Fig. 3B). Furthermore, overexpression of let-7c had no effect on the mRNA level of BCL2L1 (Fig. 3C). By contrast, forced expression of let-7c markedly decreased the protein level of BCL2L1 in CRC cells compared with the untransfected control group (Fig. 3D and E). These results indicated that BCL2L1 is a direct target of let-7c, and that let-7c regulates BCL2L1 in a post-transcriptional manner.
A

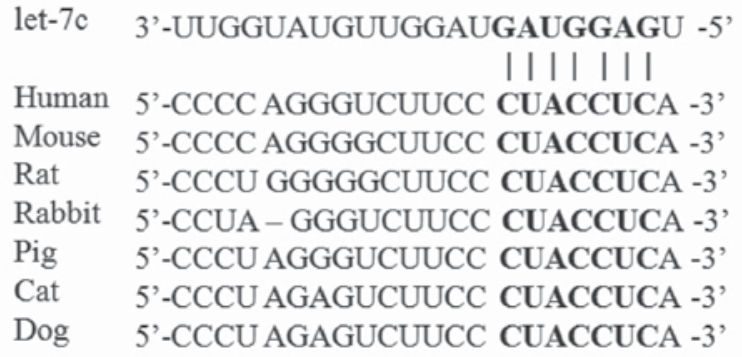

B

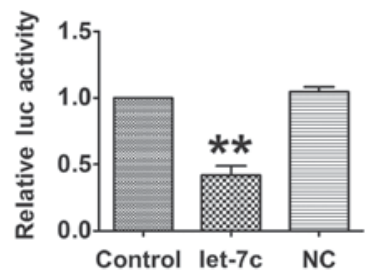

D
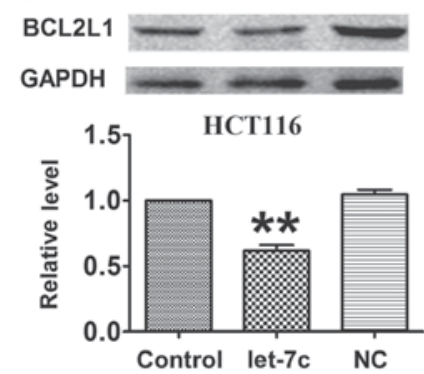

C $\square$ Control 圈 let-7c 目 NC

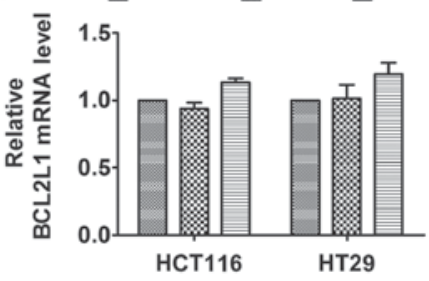

E
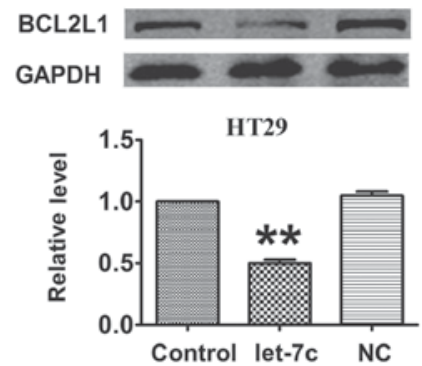

Figure 3. Let-7c regulates BCL2L1 in an post-transcriptional manner. (A) Sequences of the unique conserved microRNA-mRNA complementary sites in let-7c and BCL2L1. (B) Compared with the control group, overexpression of let-7c decreased the luciferase activity of the wild-type BCL2L1 3'UTR-containing reporter, whereas the NC showed no significant effect. (C) RT-qPCR showed that let-7c had no effect on BCL2L1 mRNA expression in HCT116 and HT29 cells. Western blot analysis showed that overexpression of let-7c inhibited the protein levels of BCL2L1 in (D) HCT116 and (E) HT29 cells. n=5 independent experiments; ${ }^{* *} \mathrm{P}<0.01$ vs. control. BCL2L1, Bcl-2-like 1; UTR, untranslated region; luc, luciferase; $\mathrm{NC}$, negative control (mimic control). 
Silencing of Lin28 promotes apoptosis via upregulating let-7c in CRC cells. As a well-known RNA-binding protein, Lin28 has been demonstrated to participate in various disease processes, including cancer and lung fibrosis, by inhibiting the biogenesis of let-7 $(13,14)$. In addition, Lin28 also contributes to the initiation and invasion of CRC (10). Therefore, we hypothesized that silencing of Lin28 may promote apoptosis in CRC by upregulating let-7c. To address this, siRNAs were transfected into cells to inhibit the expression of Lin28 (Fig. 4A), revealing that silencing of Lin28 increased the expression of let-7c in CRC cells (Fig. 4B). Furthermore, silencing of Lin28 decreased CRC cell viability, whereas inhibition of let-7c attenuated this effect (Fig. 4C and D). Further experiments showed that silencing Lin28 promoted apoptosis in CRC cells, as determined by measuring the activation of caspase- 3 (Fig. 4E and F) and the dysregulation of Bcl-2 and Bax protein (Fig. 4G and H). Knockdown of let-7c mitigated the proapoptotic effect of Lin 28 inhibition (Fig. 4E-H). These findings suggest that silencing of Lin28 induced apoptosis in CRC cells by upregulating let-7c.

\section{Discussion}

In the present study, it was demonstrated that Lin28 was upregulated and let-7c was downregulated in CRC tissues and cell lines. The forced expression of let-7c resulted in decreased cell viability and promoted apoptosis in CRC cells. Furthermore, it was demonstrated that BCL2L1 is a direct target of let-7c and may act to mediate its proapoptotic effect. Additionally, silencing of Lin 28 decreased viability and promoted apoptosis in CRC cells, whereas the knockdown of let-7c attenuated the proapoptotic action of Lin28 inhibition. Taken together, the findings demonstrate the dysregulation of the Lin28/let-7c axis in the progression of $\mathrm{CRC}$, and indicated that silencing of Lin 28 can promote apoptosis in CRC, which is mediated, at least in part, by increasing the expression of let-7c. Therefore, targeting Lin28/let-7c could be a novel strategy for the treatment of CRC.

Increasing numbers of studies have provided strong evidence thatmiRNAs contribute to various diseases, including cancer, cardiovascular diseases and diabetes (15-17). As the earliest miRNA to be discovered, let-7 is crucial in the progress of numerous diseases, particularly in various types of cancer $(8,18,19)$. A study by Trang et al $(20)$ demonstrated that loss of let-7 function enhanced lung tumor formation in vivo, whereas forced expression of let-7 significantly decreased the tumor burden in mice, indicating the inhibitory function of let-7 in cancer. Xia et al (21) found that low expression of let-7 may be a biomarker predicting poor prognosis in patients with various cancers, particularly lung cancer. In addition, Ghanbari et al (22) suggested that underexpressed let-7a-5p and let-7f-5p in plasma and stool samples from patients could serve as potential biomarkers for the early detection of CRC. Han et al (23) reported that let-7c inhibited the metastasis of $\mathrm{CRC}$ through the regulation of matrix metalloproteinase-11 and PBX homeobox 3. However, the underlying molecular mechanisms of let-7 in apoptosis, particularly during cancer, are not well understood, despite several studies reporting on the effect of let-7 on apoptosis $(24,25)$. In the present study, the results further confirmed the proapoptotic action of let- $7 \mathrm{c}$
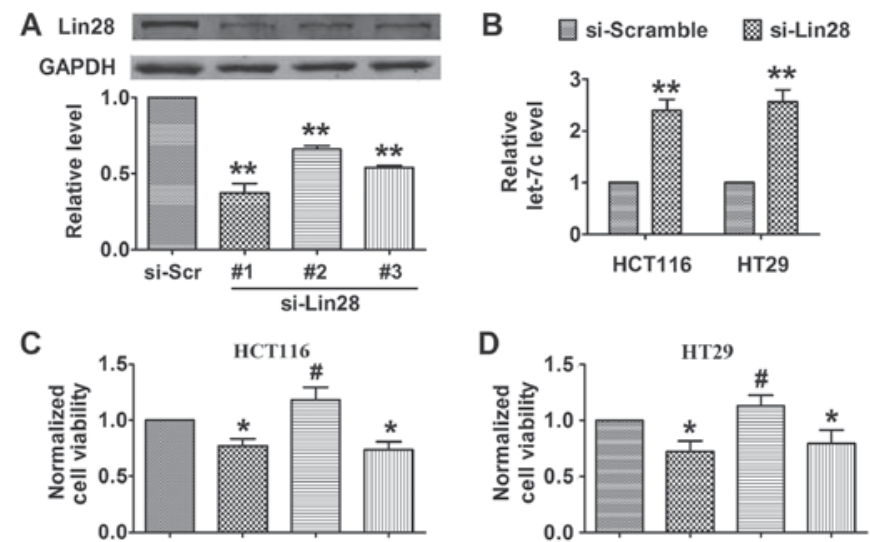

E

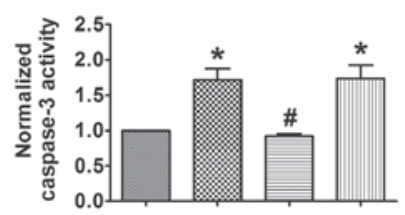

$\mathbf{F}$

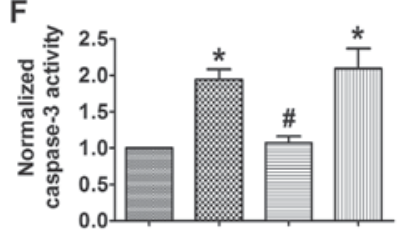

G

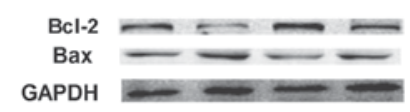

H
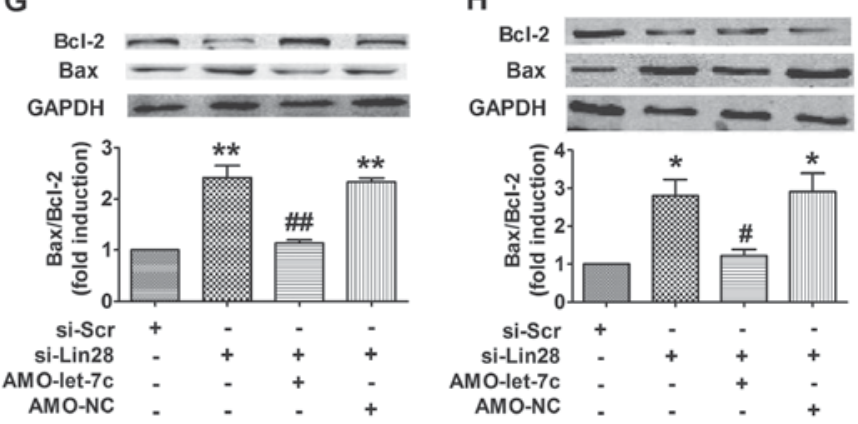

Figure 4. Inhibition of Lin28 leads to apoptosis by upregulation of let-7c in CRC cells. (A) Western blot was applied to determine Lin28 protein level in CRC cells after transfection with si-Lin28. (B) Silencing of Lin28 led to upregulation of let-7c in HCT116 and HT29 cells. Knockdown of Lin28 induced decreased cell viability in (C) HCT116 and (D) HT29 cells, whereas inhibition of let-7c mitigated the inhibitory effect of Lin28 knockdown. (E and F) Inhibition of let-7c attenuated the si-Lin28-induced activation of caspase-3 in (E) HCT116 and (F) HT29 cells. (G and H) Silencing of let-7c reversed the Lin28 inhibition-induced dysregulation of $\mathrm{Bcl}-2$ and $\mathrm{Bax}$ proteins in $(\mathrm{G})$ HCT116 and $(\mathrm{H})$ HT29 cells. $\mathrm{n}=5$ independent experiments, ${ }^{*} \mathrm{P}<0.05$ and ${ }^{* *} \mathrm{P}<0.01$ vs. si-Scramble; ${ }^{\#} \mathrm{P}<0.05$ and ${ }^{\# \#} \mathrm{P}<0.01$ vs. si-Lin 28 . CRC, colorectal cancer; siRNA, small interfering RNA; si-Scr/si-Scramble, scramble siRNA; si-Lin28, siRNA targeting Lin28; AMO-let-7c, let-7c inhibitor; AMO-NC, negative control for let-7c inhibitor.

by demonstrating the ability of let-7c to induce apoptosis in CRC cells through repressing the expression of BCL2L1; to the best of our knowledge, this is the first report of such findings. The results suggest that let-7c may be used for the treatment of CRC.

Bcl-2 family proteins serve critical roles in the regulation of the intrinsic apoptosis pathway. The activation of Bax, a key member of the Bcl-2 family, is able to promote cytochrome $c$ release and mitochondrial fission, which leads to apoptosome formation and caspase-3 activation, in turn promoting apoptosis (26). It has been reported that BCL2L1 is able to stabilize the mitochondrial localization of Bax while maintaining it in an inactive state (27). We speculate that the overexpression of let-7c results in the activation of Bax by targeting BCL2L2, and which increases cytochrome $c$ release and promotes apoptosis in CRC. 
A number of studies have demonstrated that the biogenesis of let-7 is tightly controlled by Lin 28 , which, conversely, is a direct target of let-7 $(28,29)$. Certain reports have demonstrated that the Lin28/let-7 loop influences numerous biological processes, including proliferation, differentiation, stem cell regeneration and cell aging $(11,30)$. In a recent study, Chien et al (31) revealed that Lin28/let-7 promoted the transformation of oral squamous cell carcinoma cells into cancer stem-like cells by regulating Oct $4 / \operatorname{Sox} 2$ via modulation of AT-rich interaction domain 3B and high mobility group AT-hook 2. Other studies showed that Lin28/let-7 promoted cancer progression and metastasis via regulating epithelial-mesenchymal transition (EMT) $(32,33)$ : Liu et al (32) demonstrated that Lin 28 may induce EMT via downregulation of let-7a in breast cancer cells; and Fu et al (33) reported that miR-26a suppresses tumor growth and metastasis by targeting Lin28B/let-7. Consistent with this result, a recent study by Liang et al (12) demonstrated that miR-26a inhibits lung fibrosis by repressing EMT via disrupting the Lin28B/let-7d axis. However, the role of the Lin28/let-7 axis in the process of apoptosis has not been well established.

In summary, the present study demonstrated dysregulation of the Lin28/let-7c axis in CRC patients, and further confirmed that inhibiting this axis promoted apoptosis in CRC cells by suppressing BCL2L1 through increasing the expression of let-7c. Therefore, this axis may be a novel therapeutic target for the treatment of patients with CRC. Notably, Roos et al (34) identified that N-methyl-N-[3-(3-methyl[1,2,4] triazolo[4,3-b]pyridazin-6-yl)phenyl]acetamide, which blocks the Lin28/let-7 interaction, rescued let-7 processing and function, and induced differentiation of mouse embryonic stem cells, eventually reducing tumor-sphere formation in 22Rv1 human prostate carcinoma and Huh7 human hepatocellular carcinoma cells. These findings may represent a new direction for the treatment of CRC. More studies must be performed to further explore small-molecule inhibitors of Lin28 and evaluate their therapeutic potential in CRC.

\section{Acknowledgements}

This study was supported by the Scientific Research Fund of Heilongjiang Provincial Education Department (grant no. 12541368) and Scientific Research of the Health and Family Planning Commission of Heilongjiang Province of China (grant no. 2013045).

\section{References}

1. Sforza V, Martinelli E, Ciardiello F, Gambardella V, Napolitano S, Martini G, Della Corte C, Cardone C, Ferrara ML, Reginelli A, et al: Mechanisms of resistance to anti-epidermal growth factor receptor inhibitors in metastatic colorectal cancer. World J Gastroenterol 22: 6345-6361, 2016.

2. Ciasca G, Papi M, Minelli E, Palmieri V and De Spirito M: Changes in cellular mechanical properties during onset or progression of colorectal cancer. World J Gastroenterol 22: 7203-7214, 2016.

3. De Greef K, Rolfo C, Russo A, Chapelle T, Bronte G, Passiglia F, Coelho A, Papadimitriou K and Peeters M: Multisciplinary management of patients with liver metastasis from colorectal cancer. World J Gastroenterol 22: 7215-7225, 2016.
4. Sanz-Garcia E, Grasselli J, Argiles G, Elez ME and Tabernero J: Current and advancing treatments for metastatic colorectal cancer. Expert Opin Biol Ther 16: 93-110, 2016.

5. Yin TF, Wang M, Qing Y, Lin YM and Wu D: Research progress on chemopreventive effects of phytochemicals on colorectal cancer and their mechanisms. World J Gastroenterol 22: 7058-7068, 2016.

6. Arora H, Qureshi R, Rizvi MA, Shrivastava S and Parihar MS: Study of apoptosis-related interactions in colorectal cancer. Tumour Biol 37: 14415-14425, 2016.

7. Behbahani GD, Ghahhari NM, Javidi MA, Molan AF, Feizi N and Babashah S: MicroRNA-mediated post-transcriptional regulation of epithelial to mesenchymal transition in cancer. Pathol Oncol Res 23: 1-12, 2017.

8. Sun X, Liu J, Xu C, Tang SC and Ren H: The insights of Let-7 miRNAs in oncogenesis and stem cell potency. J Cell Mol Med 20: 1779-1788, 2016.

9. Nguyen LH and Zhu H: Lin28 and let-7 in cell metabolism and cancer. Transl Pediatr 4: 4-11, 2015.

10. King CE, Cuatrecasas M, Castells A, Sepulveda AR, Lee JS and Rustgi AK: LIN28B promotes colon cancer progression and metastasis. Cancer Res 71: 4260-4268, 2011.

11. Tu HC, Schwitalla S, Qian Z, LaPier GS, Yermalovich A, $\mathrm{Ku} \mathrm{YC}$, Chen SC, Viswanathan SR, Zhu H, Nishihara R, et al: LIN28 cooperates with WNT signaling to drive invasive intestinal and colorectal adenocarcinoma in mice and humans. Genes Dev 29: 1074-1086, 2015.

12. Liang H, Liu S, Chen Y, Bai X, Liu L, Dong Y, Hu M, Su X, Chen Y, Huangfu L, et al: miR-26a suppresses EMT by disrupting the Lin28B/let-7d axis: Potential cross-talks among miRNAs in IPF. J Mol Med (Berl) 94: 655-665, 2016.

13. Livak KJ and Schmittgen TD: Analysis of relative gene expression data using real-time quantitative PCR and the 2(-Delta Delta C(T)) Method. Methods 25: 402-408, 2001.

14. Wang H, Zhao Q, Deng K, Guo X and Xia J: Lin28: An emerging important oncogene connecting several aspects of cancer. Tumour Biol 37: 2841-2848, 2016.

15. Piletič K and Kunej T: MicroRNA epigenetic signatures in human disease. Arch Toxicol 90: 2405-2419, 2016.

16. Sethupathy P: The promise and challenge of therapeutic MicroRNA silencing in diabetes and metabolic diseases. Curr Diab Rep 16: 52, 2016.

17. Shen E, Diao X, Wei C, Wu Z, Zhang L and Hu B: MicroRNAs target gene and signaling pathway by bioinformatics analysis in the cardiac hypertrophy. Biochem Biophys Res Commun 397: 380-385, 2010.

18. Bao MH, Feng X, Zhang YW, Lou XY, Cheng Y and Zhou HH: Let-7 in cardiovascular diseases, heart development and cardiovascular differentiation from stem cells. Int J Mol Sci 14: 23086-23102, 2013.

19. Li X, Wang B, Cui H, Du Y, Song Y, Yang L, Zhang Q, Sun F, Luo D, Xu C, et al: let-7e replacement yields potent anti-arrhythmic efficacy via targeting beta 1-adrenergic receptor in rat heart. J Cell Mol Med 18: 1334-1343, 2014.

20. Trang P, Medina PP, Wiggins JF, Ruffino L, Kelnar K, Omotola M, Homer R, Brown D, Bader AG, Weidhaas JB and Slack FJ: Regression of murine lung tumors by the let-7 microRNA. Oncogene 29: 1580-1587, 2010.

21. Xia Y, Zhu Y, Zhou X and Chen Y: Low expression of let-7 predicts poor prognosis in patients with multiple cancers: A meta-analysis. Tumour Biol 35: 5143-5148, 2014.

22. Ghanbari R, Mosakhani N, Sarhadi VK, Armengol G, Nouraee N, Mohammadkhani A, Khorrami S, Arefian E, Paryan M, Malekzadeh R and Knuutila S: Simultaneous underexpression of let-7a-5p and let-7f-5p microRNAs in plasma and stool samples from early stage colorectal carcinoma. Biomark Cancer 7 (Suppl 1): S39-S48, 2016.

23. Han HB, Gu J, Zuo HJ, Chen ZG, Zhao W, Li M, Ji DB, Lu YY and Zhang ZQ: Let-7c functions as a metastasis suppressor by targeting MMP11 and PBX3 in colorectal cancer. J Pathol 226: 544-555, 2012.

24. Wang T, Han P, He Y, Zhao C, Wang G, Yang W, Shan M, Zhu Y, Yang C, Weng M, et al: Lin28A enhances chemosensitivity of colon cancer cells to 5 -FU by promoting apoptosis in a let-7 independent manner. Tumour Biol 37: 7657-7665, 2016.

25. Geng L, Zhu B, Dai BH, Sui CJ, Xu F, Kan T, Shen WF and Yang JM: A let-7/Fas double-negative feedback loop regulates human colon carcinoma cells sensitivity to Fas-related apoptosis. Biochem Biophys Res Commun 408: 494-499, 2011. 
26. Youle RJ and Strasser A: The BCL-2 protein family: Opposing activities that mediate cell death. Nat Rev Mol Cell Biol 9: 47-59, 2008.

27. Renault TT, Teijido O, Antonsson B, Dejean LM and Manon S: Regulation of Bax mitochondrial localization by Bcl-2 and Bcl-x (L): Keep your friends close but your enemies closer. Int J Biochem Cell Biol 45: 64-67, 2013.

28. McDaniel K, Hall C, Sato K, Lairmore T, Marzioni M, Glaser S, Meng F and Alpini G: Lin28 and let-7: Roles and regulation in liver diseases. Am J Physiol Gastrointest Liver Physiol 310 : G757-G765, 2016.

29. Huang Y: A mirror of two faces: Lin28 as a master regulator of both miRNA and mRNA. Wiley Interdiscip Rev RNA 3 : 483-494, 2012

30. Jun-Hao ET, Gupta RR and Shyh-Chang N: Lin28 and let-7 in the metabolic physiology of aging. Trends Endocrinol Metab 27: 132-141, 2016.

31. Chien CS, Wang ML, Chu PY, Chang YL, Liu WH, Yu CC Lan YT, Huang PI, Lee YY, Chen YW, et al: Lin28B/Let-7 regulates expression of Oct4 and Sox 2 and reprograms oral squamous cell carcinoma cells to a stem-like state. Cancer Res 75 : $2553-2565,2015$.
32. Liu Y, Li H, Feng J, Cui X, Huang W, Li Y, Su F, Liu Q, Zhu J, Lv X, et al: Lin 28 induces epithelial-to-mesenchymal transition and stemness via downregulation of let-7a in breast cancer cells. PLoS One 8: e83083, 2013.

33. Fu X, Meng Z, Liang W, Tian Y, Wang X, Han W, Lou G, Wang X, Lou F, Yen Y, et al: miR-26a enhances miRNA biogenesis by targeting Lin28B and Zcchc11 to suppress tumor growth and metastasis. Oncogene 33: 4296-4306, 2014.

34. Roos M, Pradère U, Ngondo RP, Behera A, Allegrini S, Civenni G, Zagalak JA, Marchand JR, Menzi M, Towbin H, et al: A small-molecule inhibitor of Lin28. ACS Chem Biol 11: 2773-2781, 2016.

This work is licensed under a Creative Commons Attribution-NonCommercial-NoDerivatives 4.0 International (CC BY-NC-ND 4.0) License. 